www.nature.com/pj

\title{
Curing kinetics, thermomechanical and microwave behaviors of PANI-doped BSA/epoxy resin composites
}

\author{
Belkacem Belaabed $^{1}$, Saad Lamouri ${ }^{1}$ and Jean Luc Wojkiewicz ${ }^{2,3}$ \\ This work focuses on the elaboration and characterization of conducting polymer composites based on polyaniline (PANI) \\ dispersed in an epoxy resin (ER) matrix and cured using an anhydride without a catalyst. PANI-doped benzene sulfonic acid \\ was successfully synthesized in this manner and used as conducting filler. The effect of heating rate and PANI loading level on \\ the curing process was investigated by differential scanning calorimetry in the dynamic mode. Furthermore, the phase structure, \\ viscoelastic behavior and hardness of the composites were explored by dynamic mechanical thermal analysis and Vickers tests. \\ The structure and morphology of the samples were investigated by scanning electron microscopy, which revealed behavior \\ that varied as a function of the concentration of PANI in the composites. Dielectric properties were investigated up to $1 \mathrm{MHz}$. \\ The electromagnetic shielding effectiveness of the composites was measured in the microwave band from 8 to $18 \mathrm{GHz}$. \\ It was found that the electrical properties of PANI were influenced by elevated curing temperature of the ER, a variable that \\ significantly affected the composite characteristics. In addition, the dielectric properties, curing parameters, viscoelasticites \\ and electromagnetic behaviors of the composites were affecting by increasing the concentration of PANI filler in the matrix. \\ Polymer Journal (2011) 43, 683-691; doi:10.1038/pj.2011.46; published online 8 June 2011
}

Keywords: conducting polymer composite; electromagnetic shielding effectiveness; epoxy resin; polyaniline; thermomechanical

\section{INTRODUCTION}

In recent decades, the topic of conducting composite materials has attracted considerable attention, both in academic fields and in industry, because of the low cost of these materials and a growing demand for them in advanced technology and electronic system applications. ${ }^{1,2}$ Advanced composite materials with high-dielectric constants exhibit electromagnetic properties that make them suitable as electromagnetic absorbers and shielding materials to avoid electromagnetic pollution. ${ }^{3,4}$ Composites obtained through chemical and physical dispersion processes exhibit dramatically improved properties compared with pure polymers. They are used in the form of thin layers of dense materials containing various products with high-electric permittivity and/or magnetic permeability dispersed in a polymeric matrix. However, these products are considered dense and heterogeneous disordered systems. ${ }^{5}$ As a consequence, the final product has poor mechanical properties and contributes to a corrosion phenomenon, thus reducing the conductivity of the material and requiring expensive maintenance. PANI is a highly attractive replacement for these fillers; it is one of the most intensively studied intrinsically conducting polymers because of its environmental/chemical stability and good electric and electromagnetic properties. ${ }^{6,7}$ However, because of the poor mechanical properties of PANI, it is necessary to blend it with a suitable matrix. ${ }^{8}$ The dispersion of PANI filler in an insulating polymer changes the dielectric constant of the latter and produces a composite with a very high capacity to absorb or reflect electromagnetic radiation. ${ }^{9}$ Epoxy resins (ERs) are thermosetting materials that require a curing treatment to attain suitable physical and mechanical properties for industrial applications. ERs cured using amines, Lewis acids and anhydrides are widely used as coatings, adhesives and insulation in industry, because of their excellent mechanical properties, including high modulus and cohesiveness compared with other materials. ${ }^{10}$ However, using amine curing agents results in non-conductive blends. Specifically, the nucleophilic and basic lone pair electrons on the nitrogen atom of the amine group can deprotonate the conductive PANI salt. ${ }^{11}$ ERs cured using anhydrides with catalyst usually have better properties than resins cured using amines. They are less poisonous, display a higher glass transition temperature, are less hydroscopic and participate in weaker exothermic reactions. ${ }^{12}$ New materials based on conducting polymer composites can combine the high-performance mechanical properties of ER with the electrical properties of PANI. Thus, features and properties of ER composites not only depend on their chemical components and preparation procedure, but also depend on their curing conditions. Kinetic investigation is one of the most important applications of thermal analysis and has been widely conducted using isothermal or dynamic differential scanning calorimetry (DSC) technologies. ${ }^{13-15}$ Kinetic studies are especially important when fillers are used to predict performances and design curing cycles for the epoxy composites. This ensures that the cured epoxy is able to respect the requirements of its end use applications.

${ }^{1}$ Laboratory of Macromolecular Chemistry, EMP, UERCA, Algiers, Algeria; ${ }^{2}$ University Lille Nord de France, Lille, France and ${ }^{3}$ Ecole des Mines de Douai, CE, Douai, France Correspondence: B Belaabed or Professor S Lamouri, Laboratory of Macromolecular Chemistry, E.M.P, UERCA, BP. 17 EMP, BEB, Algiers 16111, Algeria. E-mail: belaabedb@yahoo.fr or lamourisaad@gmail.com

Received 15 November 2010; revised 25 March 2011; accepted 27 March 2011; published online 8 June 2011 
The aim of this study was to build on previous work $^{16}$ in which composites of PANI/ER were prepared and cured using anhydride. The PANI-doped benzene sulfonic acid (BSA) was used as conducting filler. To investigate the relationship between filler concentration and composite properties, uncured composite samples were treated at different heating rates using DSC in an inert environment. In addition, the complex modulus $\left(E^{\star}\right)$ and mechanical loss factor were measured as functions of temperature $(T)$ by dynamic mechanical thermal analysis. Furthermore, dielectric and electromagnetic behaviors were studied up to $1 \mathrm{MHz}$ and in the $\mathrm{X}$ and $\mathrm{Ku}$ bands for future applications in electromagnetic shielding (electromagnetic interference) or as radar absorbing materials.

\section{EXPERIMENTAL PROCEDURE}

\section{Materials}

Commercial medapoxy inject (ER) was obtained from Algerian Granitex (Oueud Smar, Alger, Algérie). Aniline (99\% purity), BSA, ammonium peroxydisulfate $\left(\left(\mathrm{NH}_{4}\right)_{2} \mathrm{~S}_{2} \mathrm{O}_{8}\right)$, boron trifluoride ethylamine complex $\left(\mathrm{BF}_{3}\right.$ complex, $95 \%$ purity), ethanol ( $96 \%)$, tetrahydrofuran and hexahydrophthalic anhydride (HHPA) were purchased from Sigma-Aldrich Chemie S.a.r.l. (Saint-Quentin Fallavier, France) and used without purification.

\section{Composite preparation}

PANI/ERs composites were prepared with different amounts of PANI and with or without a curing agent. PANI-doped BSA with an [oxidant]/[aniline] molar ratio of $\sim 1.15$ was prepared at $-17^{\circ} \mathrm{C}^{16}$ The product exhibited an electrical conductivity of $8-10 \mathrm{~S} \mathrm{~m}^{-1}$ as a pressed pellet. To ensure adequate dispersion, the PANI was dispersed in tetrahydrofuran for $3 \mathrm{~h}$ at room temperature using an ultrasonic bath, followed by magnetic stirring to improve the dispersion's quality. Then ER was added to the above-mentioned solution with continuous moderate stirring for $4 \mathrm{~h}$ to remove solvent residues and to ensure high dispersion and a homogeneous mixture. The weight fraction of PANI in the various composites produced was 1, 5, 10, 15, 20 and $30 \mathrm{wt} \%$. After evaporation of the solvent, the curing agent was finally added in a ratio of $81.5-100 \mathrm{~g}$ of ER. The mixture obtained was poured immediately into appropriate molds to obtain specimens for analysis, and these were subjected to a crosslinking steps in a thermostatic oven under vacuum, first at $80^{\circ} \mathrm{C}$ for $4 \mathrm{~h}$ followed by post-curing at $120^{\circ} \mathrm{C}$ for $24 \mathrm{~h}$.

\section{Techniques}

Studies on the curing kinetics of composites were performed on a NETZSCH STA 449C (204 F1) Phoenix differential scanning calorimeter (DSC, NETZSCH Instruments, Selb, Germany) operated in dynamic scanning mode from 20 to $550{ }^{\circ} \mathrm{C}$ with a single heating rate of $10^{\circ} \mathrm{C} \mathrm{min}^{-1}$ under a constant nitrogen flow of $20 \mathrm{ml} \mathrm{min}^{-1}$ to minimize oxidation of the sample during the curing process. ER was carefully mixed at the optimum ratio with a curing agent. Samples with masses of 4-8 mg were separated from the freshly prepared compositions for calorimetric measurements. Samples were placed in the aluminum calorimeter pan and then covered with an aluminum lid before undergoing a specific cure program that varied depending on the mode of measurement. The conversion degree and kinetics of the process were obtained using the instrument manufacturer's Proteus software package. Dynamic mechanical analysis was carried out using a Perkin-Elmer Pyris Diamond dynamic mechanical thermal analyzer (Perkin-Elmer, Waltham, MA, USA). The geometry of deformation was the single-cantilever bending mode. The tests were performed in the scanning temperature mode on samples with dimensions of $\sim 50 \times 8.4 \times 3.15 \mathrm{~mm}^{3}$. These samples were tested under a two-point bending condition. The runs were conducted at initial temperatures of $5-200{ }^{\circ} \mathrm{C}$ and a heating rate of $3{ }^{\circ} \mathrm{C} \mathrm{min}^{-1}$ with an oscillating frequency of $1 \mathrm{~Hz}$. Vickers tests were conducted using an HWDM-1 apparatus at room temperature to evaluate the hardness response of the materials. Morphological analysis was carried out using a Hitachi S-4300 SE/N scanning electron microscope (Hitachi, Tokyo, Japan) operating between 5 and $20 \mathrm{kV}$ and between 35 and $50 \mu \mathrm{A}$. To avoid the effects of electric charge on the surface and to facilitate the flow of electrons, samples were coated with a thin layer of gold. Electrical and dielectric measurements were conducted on circular films that were $30 \mathrm{~mm}$ in diameter with a thickness $(d)$ on the order of $1 \pm 0.05 \mathrm{~mm}$. These films were coated on both sides with a gold paste using an Agilent $4284 \mathrm{~A}$ RF impedance analyzer (Agilent Technologies, Englewood, CO, USA) with a frequency $(f)$ ranging from $20 \mathrm{~Hz}$ to $1 \mathrm{MHz}$. The direct current (DC) conductivity was calculated from the current-voltage dependence using a Signatone 1160 series probe station (Signatone Corporation, Gilroy, CA, USA) and an LCR-type Data Bridge 9341 (Tinsley Precision Instruments, Surrey, UK). The electromagnetic parameters $\left(S_{11}, S_{21}\right)$ were measured using an Agilent technologies E8362B Vector Network Analyzer (Agilent Technologies) working from $10 \mathrm{MHz}$ to $20 \mathrm{GHz}$. In this frequency domain, two sets of waveguides were used: a WR-90 for the X band and a WR- 62 for the Ku band. The wave-guides were linked to the vector network analyzer using two cables with standard APC-7 connectors.

\section{RESULTS AND DISCUSSION}

\section{DSC Study}

Thermal history has an important effect on the network architecture and final properties of thermosetting ERs. Therefore, DSC, which measures the heat flow from the reacting system as a function of temperature, can offer insights into the relation between the heat of reaction, $T_{\mathrm{g}}$, and the curing behavior. DSC is a very convenient tool for studying the overall reaction between the ER and the crosslinking agent.

All kinetic studies start with a basic rate equation:

$$
\mathrm{d} \alpha / \mathrm{d} t=k . f(\alpha)
$$

where $\alpha$ is the chemical conversion at a given time and temperature, $f(\alpha)$ is some function of the fractional conversion $\alpha$, and $\mathrm{d} \alpha / \mathrm{d} t$ is the rate of conversion. The rate constant, $k$, is usually assumed to be of the Arrhenius form:

$$
k=A \exp (-E / R T)
$$

where $A$ is a constant, $R$ is the gas constant, $E$ is the activation energy and $T$ is the absolute temperature. ${ }^{17}$

The curing reaction of ER is exothermic. The onset temperature $\left(T_{\mathrm{i}}\right)$, peak temperature $\left(T_{\mathrm{p}}\right)$, end temperature $\left(T_{\mathrm{f}}\right)$ and heat flow of the curing reaction can be obtained from DSC thermograms. The reaction was considered complete when the rate curve leveled off to a baseline. The total heat of reaction was estimated by drawing a straight line connecting the baseline before and after the peak and integrating the area between the peak and this line. The conversion degree was defined as $0 \%$ at the beginning of the exothermic peak in the DSC curve and $100 \%$ at the end of the exothermic peak. To evaluate the effect of PANI concentration on curing parameters, DSC investigations of composites with and without hardener or catalyst were conducted. In addition to the single heating rate, measurements were also made at different heating rates of 5, 10, 15, 20, 25 and $30^{\circ} \mathrm{C}$. Typical DSC curves displaying heat flow as a function temperature are shown in Figures 1 and $3 \mathrm{a}-\mathrm{c}$.

Figure 1 shows that the exothermic curing peak of the ER/HHPA/ $\mathrm{BF}_{3}$ appears at $120^{\circ} \mathrm{C}$, corresponding to the complete crosslinking reaction of the ER. The autocatalytic peak of the ER peak appears at $375^{\circ} \mathrm{C}$. However, for the ER/HHPA system with and without PANI, the exothermic curing peak appears at 210 and $242^{\circ} \mathrm{C}$, respectively, whereas in the case of ER/PANI, the peak appears at $272{ }^{\circ} \mathrm{C}$. These observations are in agreement with the thermogram obtained by thermogravimetric analysis (Figure 2). ${ }^{16}$ The evaporation and degradation of BSA and the oxidation process of the PANI structure and its composites occurred between 170 and $390^{\circ} \mathrm{C} .^{7,18,19}$ 


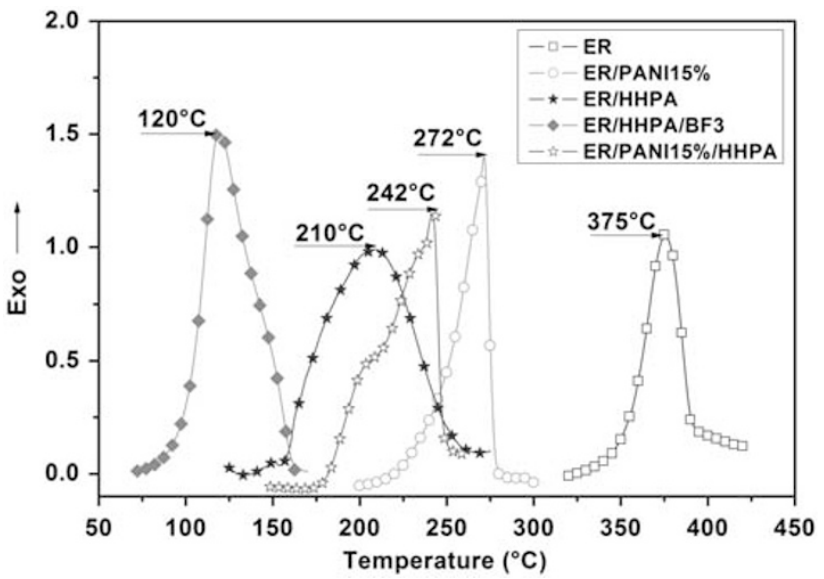

Figure 1 Differential scanning calorimetry thermograms of different epoxy resin (ER) composites. HHPA, hexahydrophthalic anhydride; PANI, polyaniline. A full color version of this figure is available at Polymer Journal online.

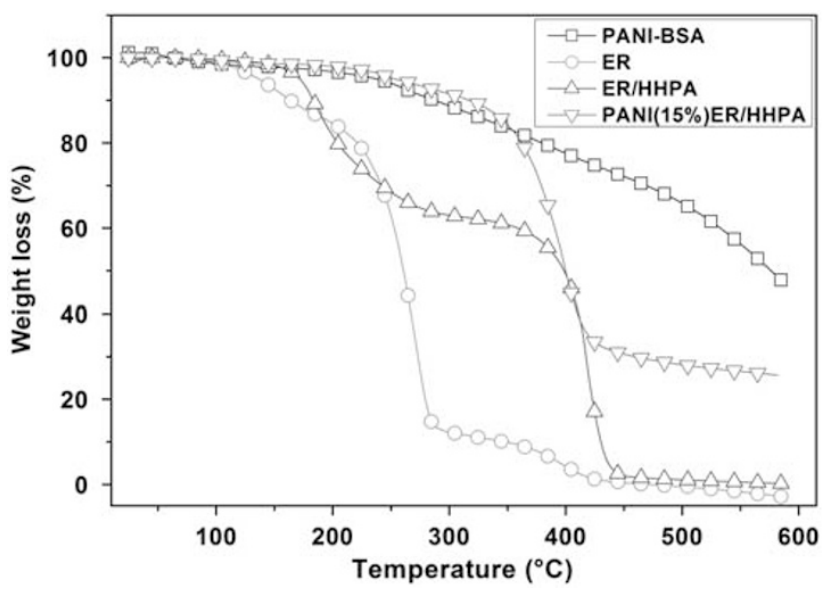

Figure 2 Thermograms of thermogravimetric analysis of polyaniline-doped benzene sulfonic acid (PANI-BSA) and cured composites with and without hexahydrophthalic anhydride (HHPA). ${ }^{16}$ ER, epoxy resin. A full color version of this figure is available at Polymer Journal online.

To avoid deprotonation of the conducting PANI and maintain the conductivity of composites after the curing process, it was imperative to add a catalyst.

In all following investigations, composites prepared without a catalyst were examined and characterized to investigate the effects of PANI loading on the characteristics of the resulting composites. Figure 3 reveals the relationship between the heat flow of curing reaction energy and peak temperatures, and the PANI content in the ER matrix with and without a curing agent.

All thermograms of composites with a hardener exhibited one exothermic peak accompanied by a small shoulder. In this case, the heat flow increased with increasing PANI concentration in the matrix for both systems with and without a hardener. However, the curing peak temperature decreased with increasing PANI loading in all composites (Figure 3c). It decreased from 259 to $202^{\circ} \mathrm{C}$ in composites with a curing agent, but varied from 291 to $262{ }^{\circ} \mathrm{C}$ in composites without a hardener. This may be due to the double action of both the PANI and the HHPA as curing systems. At higher temperatures, PANI-doped BSA can function as an amine hardener. ${ }^{5}$ These results showed that the PANI-doped BSA affected the curing reaction of the
ER at high temperatures. Consequently, the displacement of the peak temperature to lower values with increasing PANI loading in the matrix may be due to the reaction between PANI and the ER. The reaction between epoxy and the amine groups of PANI may be characterized by the shoulder (Figure $3 b$ ).

According to Kissinger's approach, the kinetic parameters for the curing reaction of PANI/ER composites can be evaluated by the expression: ${ }^{18,20}$

$$
\ln \left(\beta / T_{\mathrm{p}}^{2}\right)=\ln \left(A R / E_{\mathrm{a}}\right)+\left(-E_{\mathrm{a}} / R T_{\mathrm{p}}\right)
$$

where $\beta$ is the heating rate $\left(\mathrm{K}^{\circ} \min ^{-1}\right), T_{\mathrm{p}}$ is the absolute peak temperature, $E_{\mathrm{a}}$ is the activation energy $\left(\mathrm{kcal} \mathrm{mol}^{-1}\right)$ and $R=1.98 \mathrm{cal} \mathrm{K}^{\circ} \mathrm{mol}^{-1}$ is the gas constant.

$$
E_{\mathrm{a}} / R=\mathrm{d}\left(\ln \left(\beta / T_{\mathrm{p}}^{2}\right)\right) / \mathrm{d}\left(1 / T_{\mathrm{p}}\right)
$$

By plotting $\ln \left(\beta / T_{\mathrm{p}}{ }^{2}\right)$ as a function of $\left(1 / T_{\mathrm{p}}\right)$, we obtained the activation energy.

A PANI (15\%)/ER composite with a curing agent near the percolation threshold was selected to evaluate the curing kinetics in dynamic mode at different curing rates $\left(5,10,15,20,25\right.$ and $\left.30^{\circ} \mathrm{C} \mathrm{min}^{-1}\right)$ at temperatures ranging from 20 to $480{ }^{\circ} \mathrm{C}$. Plots of the resulting Kissinger equations are shown in Figures $4 \mathrm{a}-\mathrm{e}$. Activation energies were determined based on the strong and overlapped exothermic peaks observed in the dynamic curves. The fractional conversions $(\alpha)$ as a function of temperature were obtained by integrating the DSC signal.

It was revealed that PANI (15\%)/ER/HHPA systems exhibit one exothermic peak that increases in intensity with heating rate. This peak was related to an esterification reaction between the ER and anhydride. The peak temperature $\left(T_{\mathrm{p}}\right)$ shifted to higher values with increased heating rate, jumping from 222 to $241{ }^{\circ} \mathrm{C}$. It can be seen that the isoconversional temperature also increased linearly with the heating rate at the same conversion rate (Figure $4 \mathrm{~d}$ ), indicating that reaction heat increased with the heating rate. The dependence of the activation energy $\left(E_{\mathrm{a}}\right)$, on the composites formulation was observed. The activation energy was calculated from the slopes of the curves generated by the Kissinger model (Figure $4 \mathrm{e}$ ) and had a value on the order of $37.3 \mathrm{~kJ} \mathrm{~mol}^{-1}$, is typical for reactions of epoxy composites. ${ }^{21}$ According to the esterification reactions between ER and anhydride and between epoxy and the amine-imine groups of PANI, 12,22,23 the hydroxyl groups that were formed during the curing reaction stage facilitated ring opening and could catalyze the reaction. The activation energy was nearly constant, it varied from 34 to $43 \mathrm{~kJ} \mathrm{~mol}^{-1}$, indicated that more hydroxyl groups were formed during the curing reaction stage. As the curing reaction proceeded gradually, crosslinking intensified and the molecular mobility of the epoxy system was reduced. The transition from the gelation phase to the vitrification stage $^{24,25}$ induced diffusion control, which can be recognized by the increased value of the activation energy at the final stages of conversion, as shown in Figure 4e. This observation revealed that the activation energy increased as the conversion proceeded and confirmed that vitrification occurred at an early stage in the curing reaction.

\section{Dynamic mechanical thermal analysis}

Dynamic mechanical analysis is one technique used to investigate the thermomechanical properties of composites to verify the expected influence of PANI concentration on the mechanical cure states and kinetic properties of those composites. It is also a sensitive method for detecting changes in internal molecular mobility. The structure and viscoelastic characteristic can thus be determined from the 

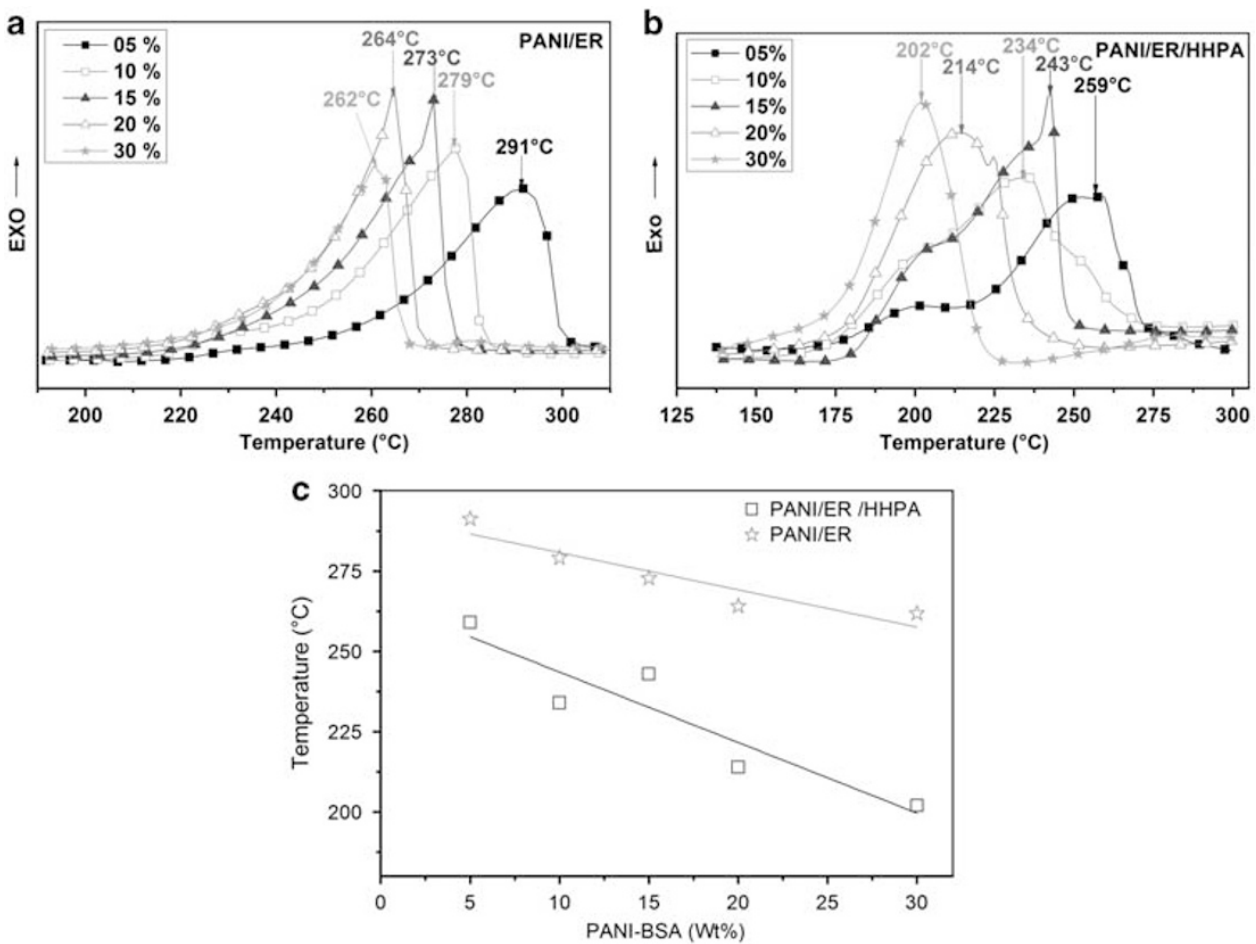

Figure 3 Differential scanning calorimetry thermograms of polyaniline (PANI)/epoxy resin (ER) with different amounts of PANI-doped benzene sulfonic acid (a) without hexahydrophthalic anhydride (HHPA), (b) with HHPA and (c) the PANI loading dependence of the peak temperature $\left(T_{\mathrm{p}}\right)$ of composites. A full color version of this figure is available at Polymer Journal online.

dynamic and loss modulus. The storage modulus is related to the tendency of the material to store or return energy when an oscillatory force is applied to the sample, and the loss modulus is related to the material's ability to dissipate the energy. These properties were measured to study the relationship between the addition of PANI-doped BSA to the ER matrix and the curing process. Initial work was conducted to ensure that the response was in the linear viscoelastic region. The storage modulus $\left(E^{\prime}\right)$ and loss modulus $\left(E^{\prime \prime}\right)$ were measured as functions of temperature at a fixed frequency of $1 \mathrm{~Hz}$. Figure 5 shows the temperature dependence of the storage modulus $\left(E^{\prime}\right)$ and the loss modulus $\left(E^{\prime \prime}\right)$ of PANI/ER/HHPA composites at different PANI concentrations.

In all figures, the storage modulus is constant up to $50{ }^{\circ} \mathrm{C}$, followed by a sudden drop at the glass transition temperature. However, the storage modulus increases as a function of increasing PANI content in matrix, indicating that there is a large mechanical fortification produced by the PANI filler over the entire temperature range and that this reinforcement is greater in the rubbery state than in the glassy state. At temperatures of $80^{\circ} \mathrm{C}$ and above, the storage modulus is relatively constant, indicating that stable crosslinked networks have formed within the composites. All system formulations showed one relaxation peak, as revealed by the loss factor, tang $(\delta)=\left(E^{\prime \prime} / E^{\prime}\right)$, corresponding to the transition temperature $\left(T_{\mathrm{g}}\right)$ of the composites, which varied between 70 and $77^{\circ} \mathrm{C}$. This variation may be explained by the increase in free volume as a function of PANI concentration, which resulted in an increase of motion ability of the rubber molecules because of the greater number of soft segments in the polymer backbones. This relaxation process is associated with the $T_{\mathrm{g}}$ phenomenon. Furthermore, below $T_{\mathrm{g}}$, the reinforcement effect on the storage modulus is large. This is not surprising, considering the amount of added filler. However, the most important factors that can affect $T_{\mathrm{g}}$ are the level of particle dispersion, the size of and spacing between particles, the degree of particle dispersion, composite homogeneity and the curing conditions, including curing speed and the degree of crosslinking. The improvement in the $T_{\mathrm{g}}$ observed in this study may arise from some of these factors. ${ }^{26,27}$

\section{Micro hardness}

Using Vickers tests, micro hardness measurements of the surfaces of PANI/ER/HHPA composites are shown in Figure 6. The hardness depends strongly on the concentration of PANI-doped BSA filler in the composites. The material becomes more rigid with increasing filler content, as expected from the addition of PANI into an epoxy matrix. In general, polymers with active fillers of micrometer size demonstrate improved hardness, but their elastic and impact properties become worse because of stress concentration from the presence of filler particles. ${ }^{1}$

\section{Morphology characterization}

The morphology of the PANI and its composites was investigated using a scanning electron microscope (SEM), and the results are shown in Figure 7.

In these images, the existence of crystal, lamellar and linear forms with flat and smooth surfaces can be observed in the PANI-BSA micrographs. This finding indicates that the doping agent used gave a pseudo-metallic characteristic to the samples. In the SEM micrographs of cured PANI/ER composite films, the white regions are PANI particles. Systems will exhibit the presence of defects and fluctuations of composition in space when the scale of observation becomes smaller and smaller. Small inhomogeneities that are well defined and well dispersed in a matrix can be described as an ordered structure on a larger scale. When a low ratio of PANI was used (Figure $7 \mathrm{~b}$ ), the 
a
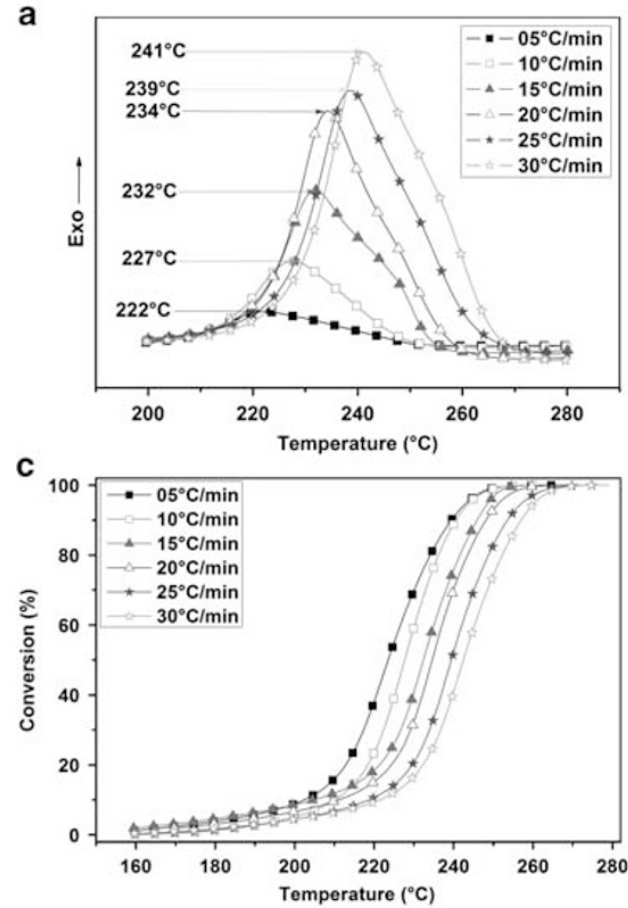

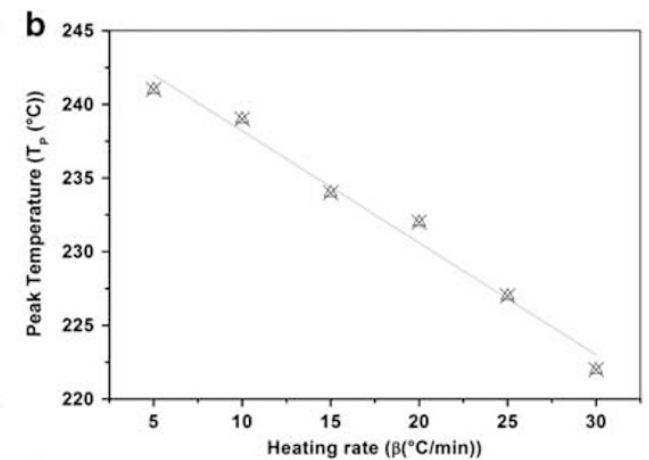

d

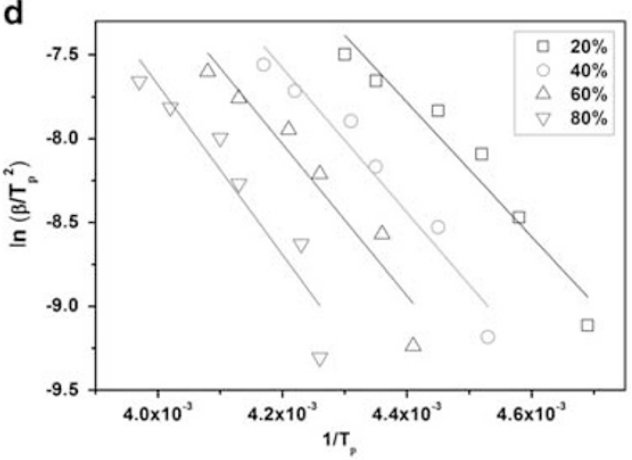

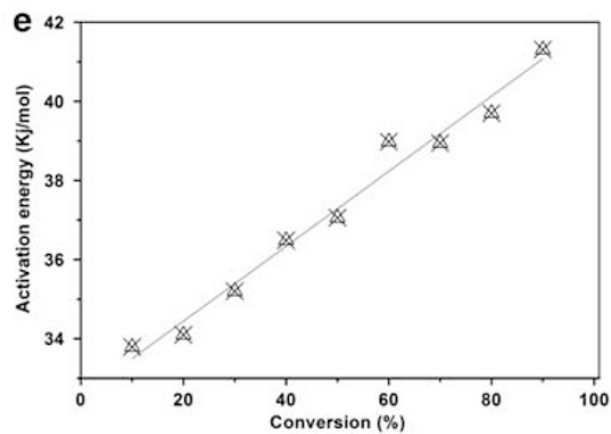

Figure 4 (a) Differential scanning calorimetry thermograms of a polyaniline (PANI)/epoxy resin/hexahydrophthalic anhydride composite (15\% PANI) at different heating rates, (b) the relationship between heating rate and peak temperature $\left(T_{\mathrm{p}}\right)$, (c) fraction conversion as a function of temperature, (d) isoconversional plots and (e) activation energy function of the conversion. A full color version of this figure is available at Polymer Journal online.

PANI particles were homogeneously and randomly dispersed in the epoxy matrix, indicating a homogeneous mixture of phases. However, when the weight content of PANI in the samples was above $15 \mathrm{wt} . \%$, agglomerates of PANI formed in the matrix. For the sample with 15\% PANI, it appears that the PANI was finely dispersed in the matrix, which could indicate that part of the PANI dissolved or reacted with the ER at higher temperatures. In addition, the existence of the PANI fillers resulted in the enlargement of the distance between the branches of the crosslinked network, a finding confirmed by DTMA investigations (Figure 5), which showed a shift in $T_{\mathrm{g}}$ to lower temperatures with increasing PANI concentration in the matrix. ${ }^{28}$

\section{Dielectric properties}

The DC conductivity of composite materials prepared without a catalyst and containing 30\% (wt \%) of PANI-doped BSA reached a maximum value of $2.38 \times 10^{-5} \mathrm{~S} \mathrm{~m}^{-1}$. At PANI loading rates lower than $5 \%$, the measured conductivities corresponded to that of the non-conducting matrix (DC conductivity: $10^{-8}-10^{-14} \mathrm{~S} \mathrm{~m}^{-1}$ ), as shown in Figure 8 . The percolation threshold was situated between 10 and $20 \%$ PANI.
The dielectric parameters and $\mathrm{AC}$ conductivity were used to obtain more detailed information on the structure of the composites. These parameters have been calculated from the capacitance ( $C$, in Farads) and conductance ( $G$, in Siemens) of the materials using the following equations: ${ }^{29-35}$

$$
\begin{aligned}
& \varepsilon^{\prime}=C_{\mathrm{p}(\text { sample })} / C_{0}, \\
& e^{\prime \prime}=G_{(\text {sample) }} \cdot d / \omega \varepsilon_{0} \cdot A, \\
& \sigma_{\mathrm{AC}}(\omega)=\varepsilon_{0} \cdot \varepsilon^{\prime \prime} \cdot \omega
\end{aligned}
$$

$\varepsilon^{\prime}, \varepsilon^{\prime \prime}$ are the real and imaginary parts of the complex dielectric constant $\varepsilon^{*}$. Tang $(\delta)=\left(\varepsilon^{\prime \prime} / \varepsilon^{\prime}\right)$ is the dissipation factor, and $\delta$ is the phase angle. $C_{0}=\left(\varepsilon_{0} A / d\right)$, is the geometrical capacitance of a vacuum of the same dimensions as the sample. $A$ and $d$ are the area and thickness, respectively, of the sample. $C_{p}$ is the capacitance, $\varepsilon_{0}=8854 \times 10^{-12} \mathrm{Fm}^{-1}$ is the permittivity of the free space, $\omega=2 \pi f$ is the angular frequency and $\sigma_{\mathrm{AC}}$ is the AC conductivity.

The dielectric behavior of samples is illustrated in Figure 9a-e. This figure shows the variation of the real part $\left(\varepsilon^{\prime}\right)$ and imaginary part $\left(\varepsilon^{\prime \prime}\right)$ 

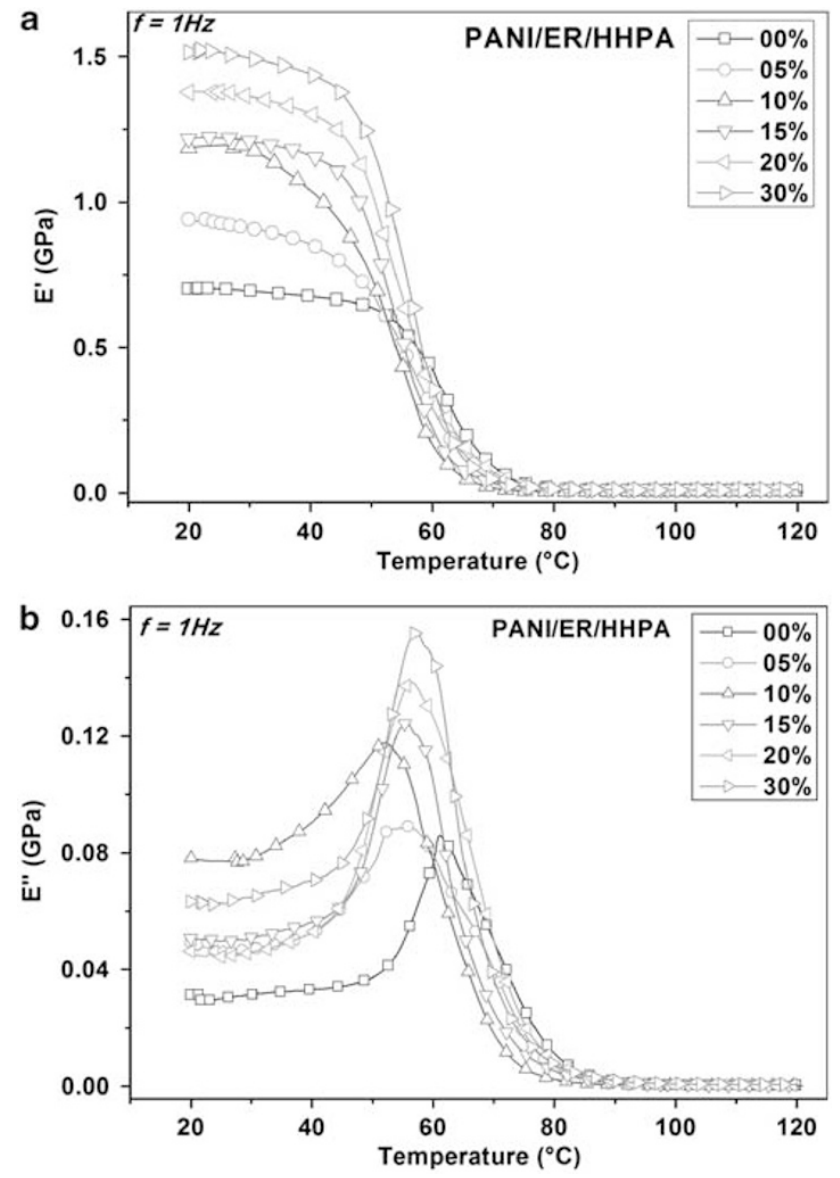

Figure 5 Thermomechanical behaviors of polyaniline (PANI)/epoxy resin (ER)/hexahydrophthalic anhydride (HHPA) composites: (a) storage modulus $\left(E^{\prime}\right)$ and $(\mathbf{b})$ loss modulus $\left(E^{\prime \prime}\right)$. A full color version of this figure is available at Polymer Journal online.

of the dielectric constant of PANI-doped BSA and the frequency dependence composites versus frequency of $\varepsilon^{\prime}, \varepsilon^{\prime \prime}$, as well as the changes in conductivity and the evolution of the real and imaginary parts of $\varepsilon^{\star}$ with increasing PANI contents in the composites at $1 \mathrm{MHz}$.

PANI-doped BSA exhibited values of 206, 2323 and 11.27 for $\varepsilon^{\prime}, \varepsilon^{\prime \prime}$ and $\operatorname{tang} \delta$, respectively, at $20 \mathrm{~Hz}$, which decreased to 162,25 and 0.15 at $1 \mathrm{MHz}$ (Figure 9a). However, in all cases, it was observed that the addition of PANI powder to the matrix initially increased the dielectric constant to high values at lower frequencies. This constant decreased slightly when the frequency increased further, maintaining linear stability, and the variation in the dielectric constant eventually became very low and almost constant with further increases in frequency. Accordingly, loss tang was also found to increase with increasing PANI loading and frequency; the loss tang was found to have a value of 0.052 at $20 \%$ (wt \%) PANI and $1 \mathrm{MHz}$. This trend could be due to a decrease in the dipolar polarization of the matrix and the accumulation of charges at the interface between the PANI particles and the ER, resulting in a large-scale field distortion. These effects could also be due to the variation in the distribution of PANI in the matrix, as revealed by SEM. In addition, the purity of some of the starting materials was not very high, and these were used without purification; thus, the elaborate composites could contain impurities from these materials. Such impurities could be the cause of the anomalies observed in the dielectric spectra at low frequencies. The observed

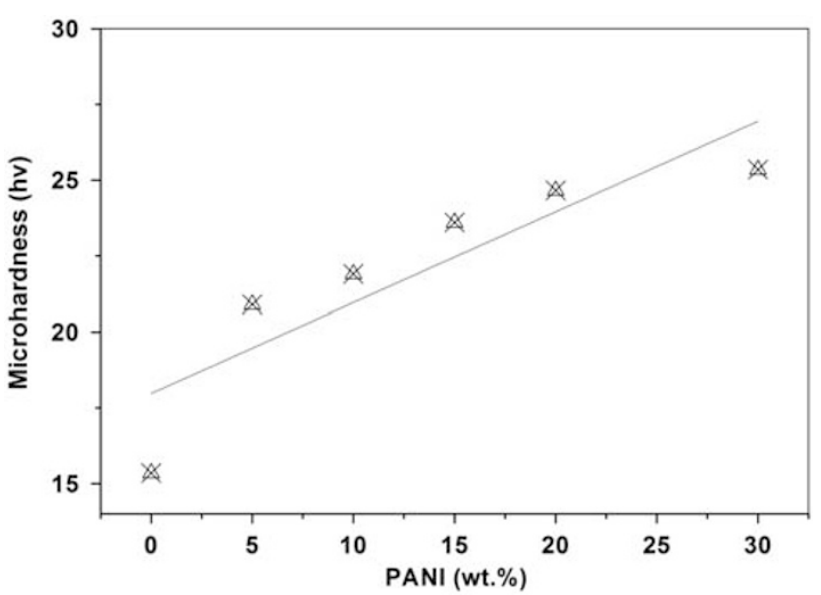

Figure 6 Microhardness behavior of polyaniline/epoxy resin/hexahydrophthalic anhydride composites.

dielectric loss behavior is in accordance with the conductivity and dielectric constant of the reported composites. It could also be the case that the large increase in $\varepsilon^{\prime \prime}$ at low frequencies is an extrinsic effect linked to a Maxwell-Wagner effect and also to the DC conductivity caused by impurities. Figure $9 \mathrm{~d}$ shows that the frequency dependence of conductivity is strongly related to the character of the phenomenon of conduction and charge transport in these composites. AC conductivity increased with increases in frequency, in accordance with Equation (7). At higher frequencies, the conductivity increased and was observed to follow Jonscher's universal power law, given by $\sigma_{\mathrm{AC}}$ $(\omega) \sim A \omega^{s}$, which describes the pseudo-linear dependence of ac conductivity on the frequency for various amorphous materials, where $A$ is a constant dependent on temperature and $(s \leqslant 1)$ is a criterion sensitive to the type of conduction-relaxation mechanism dominant in the amorphous materials. The conductivity could be caused by migrating charge carriers or it may refer to a loss of energy associated with the dispersion of $\varepsilon^{\prime} \cdot{ }^{36}$ In all graphs of these composites, the increase of AC conductivity with PANI loading may occur for two reasons. First, the increased conductivity may be the result of increased charge carrier concentration and easier formation of conducting networks resulting from better interparticle contact among PANI particles dispersed in the polymer matrix. Second, the conductivity might also increase as a result of the difference in the dielectric constants and conductivities of PANI and ER. This phenomenon appears in heterogeneous systems because of the accumulation of mobile charges at the interfaces and the formation of large dipoles on clusters. The frequency dependencies of $\varepsilon^{\prime}$ and $\varepsilon^{\prime \prime}$ up to $1 \mathrm{MHz}$ in the PANI/ER composites are shown in Figure 9e. The addition of PANI to the matrix and the elimination of agglomerates surrounded by the insulating matrix result in the formation of a fine network that makes charge transfer easy through the well-ordered polymer, leading to enhanced dielectric properties of the composites. With the application of an external electric field, strong dipole-dipole interactions between the PANI and ER allow the charges to hop from one dipole to another throughout the polymer composite matrix. As the amount of PANI increases, the dipole density increases and then enhances the mobility of the dipoles, which in turn depend on the mobility of the polymer chains to which the dipoles are attached. As a result, an increase in the permittivity value and the dielectric loss factor is observed with increased PANI loading in the composite. ${ }^{37}$ These results reveal that the addition of PANI increases the conductivity of ER composites to a 

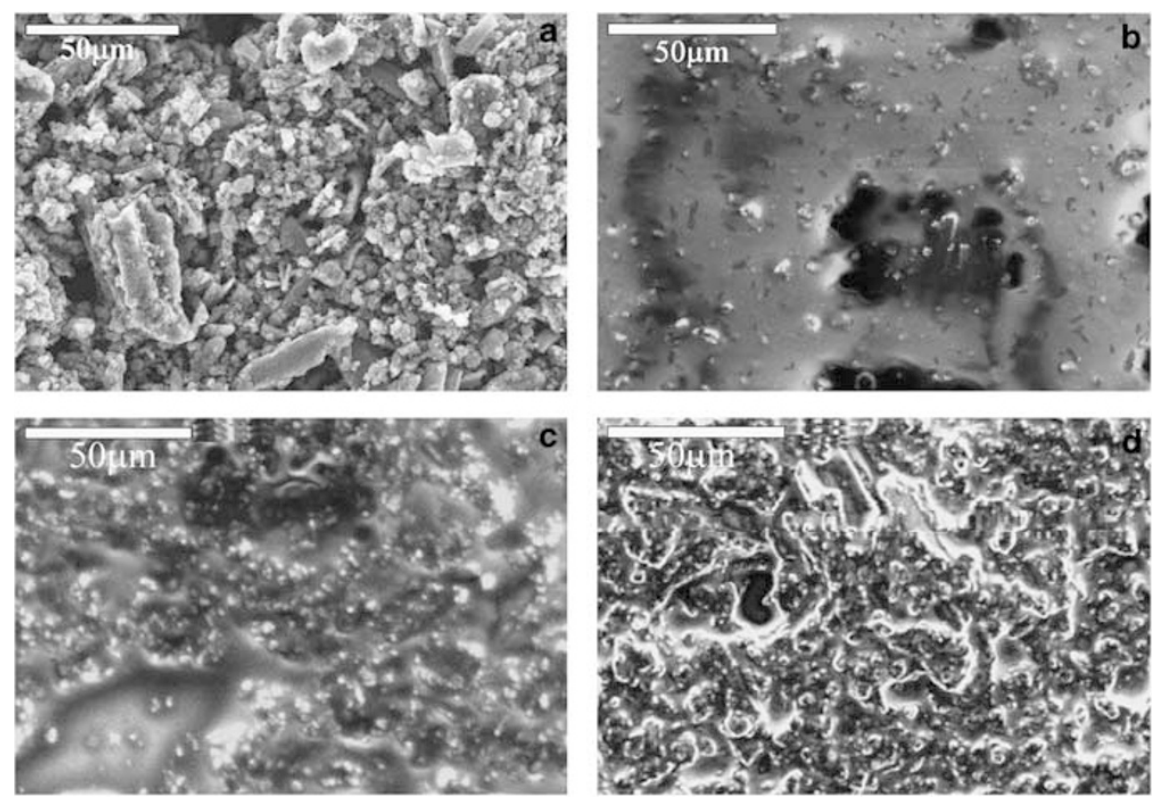

Figure 7 Scanning electron micrographs: (a) polyaniline-doped benzene sulfonic acid (PANI-BSA) powder, (b) PANI/epoxy resin/hexahydrophthalic anhydride composites containing 5\% PANI, (c) $15 \%$ PANI and (d) $20 \%$ PANI.

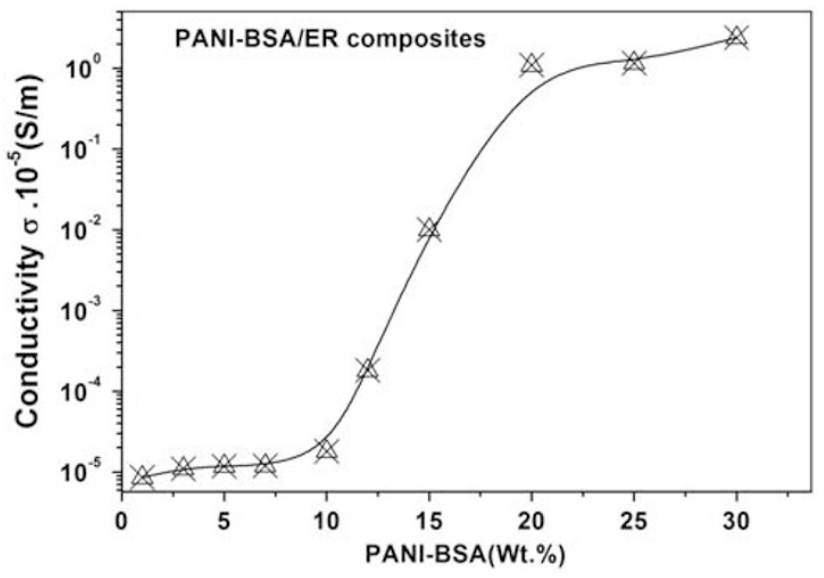

Figure 8 Polyaniline-doped benzene sulfonic acid (PANI-BSA) loading dependence of the DC conductivity of composites. ER, epoxy resin.

value that is suitable for antistatic and radar absorbing material applications. $^{38-40}$

\section{Electromagnetic shielding effectiveness}

Shielding effectiveness (SE) is typically used to assess the shielding properties of a material and is defined as the ratio of the power transmitted through the material $\left(P_{\mathrm{t}}\right)$ to the incident power $\left(P_{\mathrm{i}}\right)^{41}$

$$
\mathrm{SE}(\mathrm{dB})=-10 \log \left(P_{\mathrm{t}} / P_{\mathrm{i}}\right)
$$

For a shielding material, total $\mathrm{SE}$ is the sum of $\mathrm{SE}=\mathrm{SE}_{\mathrm{R}}+\mathrm{SE}_{\mathrm{A}}+\mathrm{SE}_{\mathrm{M}}$, where $S E_{R}$ is the shielding resulting from reflection, $S E_{A}$ is due to absorption and $\mathrm{SE}_{\mathrm{M}}$ is due to multiple reflections. Measurements of the electromagnetic parameters $\left(S_{11}, S_{21}\right)$, which represent the reflection and transmission coefficients, were carried out using a vector network analyzer from $10 \mathrm{MHz}$ to $20 \mathrm{GHz}$. Figure 10 shows the electromagnetic interference SE of the PANI/ER/HHPA at different loadings of PANI in the frequency range of $8-18 \mathrm{GHz}$.
The SE value increases with the addition of conducting polymer to the ER matrix. SE is nearly constant over the entire frequency range studied. The SE value was $\sim 6 \mathrm{~dB}$ for a sample loaded with $30 \%$ (wt $\%$ ) PANI. The increase in the SE was due to the presence of more charge carriers and to polarization or the jumping of charge from one position to another. The observed results prove that the electromagnetic parameters were also altered by the higher curing temperature, which affected electrical composites because of the partial deprotonation of PANI, as indicated by the results of the thermogravimetric analysis and DSC investigations.

\section{CONCLUSION}

In the present study, conducting PANI-doped BSA composites with DC electric conductivities of $8-10 \mathrm{~S} \mathrm{~m}^{-1}$ have been synthesized successfully by conventional oxidative polymerization. The DSC and electrical investigations show that the properties of the PANI were altered at higher curing temperatures. A homogenous dispersion of PANI in the ER was indicated by the results of SEM studies. Curing behavior of various composite formulations was also investigated. It was revealed that the curing parameters and viscoelastic behaviors of the composites were affected by the PANI loading in the matrix. The curing kinetics investigations indicated that the onset and maximum temperatures of cured composites decreased with increasing PANI in the matrix. Furthermore, the dielectric and SE constants increased with increasing PANI concentrations. The increase in dielectric constant with increasing PANI concentration may be attributed to the intrinsic electric dipole polarization and to conformational changes occurring in the PANI. With the incorporation of up to $15 \mathrm{wt} \%$ PANI in the matrix, the composites were shown to exhibit permittivity values of 8.35 at $1 \mathrm{MHz}$ and $\mathrm{SE}$ values of up to $3.5 \mathrm{~dB}$ in the microwave band. These low values were due to low electric conductivity of PANI and its deprotonation at high temperatures during the curing process. Future work will focus on the investigation and optimization of the electrical conductivity of PANI, as well as low percolation thresholds to improve absorption within composite films. The use of catalysts such as Lewis acids or Lewis bases is also 

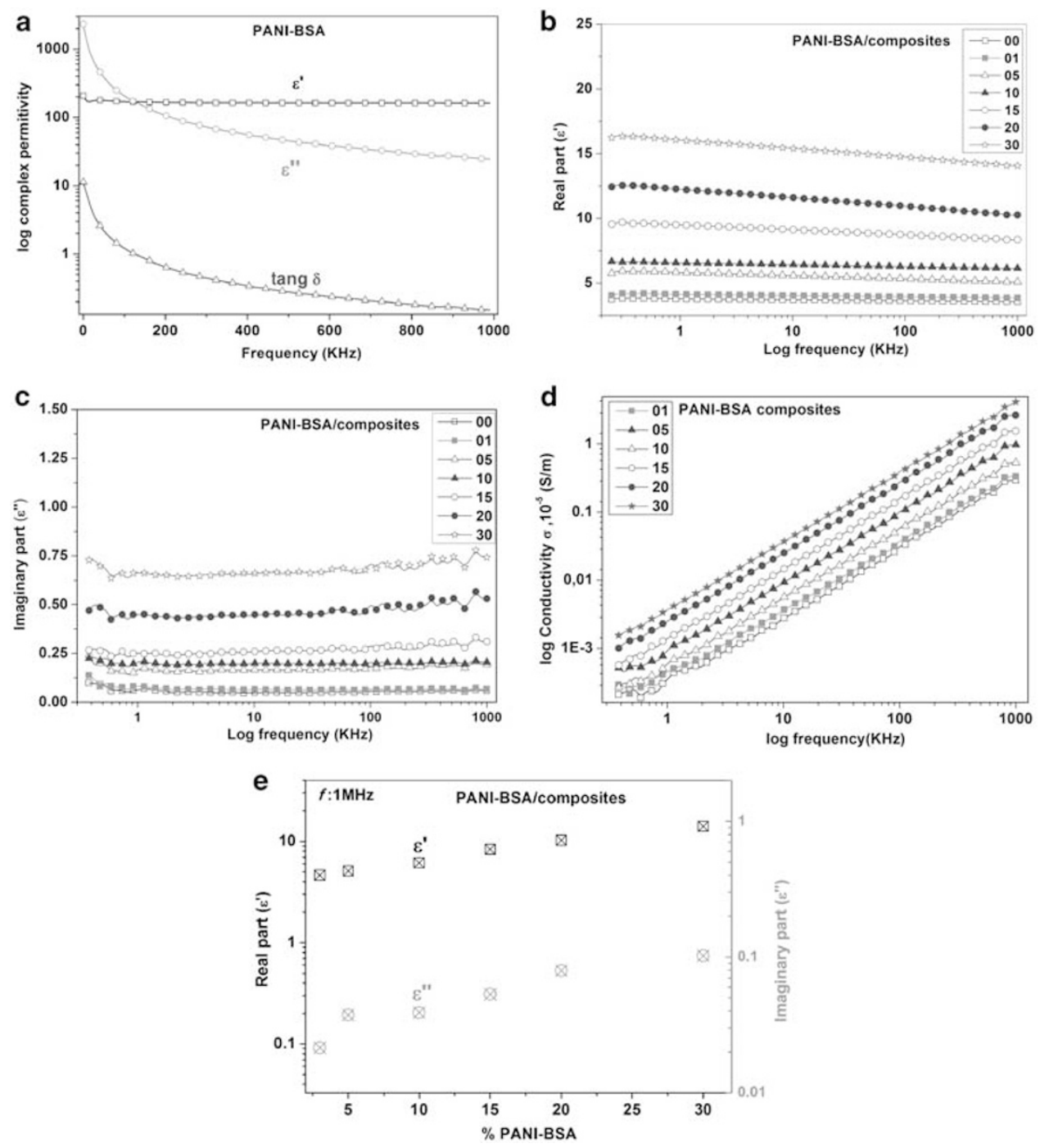

Figure 9 Frequency dependence of (a) permittivity of the polyaniline-doped benzene sulfonic acid (PANI-BSA) complex, (b) the real part of the dielectric constant, (c) the imaginary part of the dielectric constant, (d) AC conductivity $\sigma(\omega)$ and (e) PANI loading dependence of the real and imaginary parts of the dielectric constant at $1 \mathrm{MHz}$. A full color version of this figure is available at Polymer Journal online.

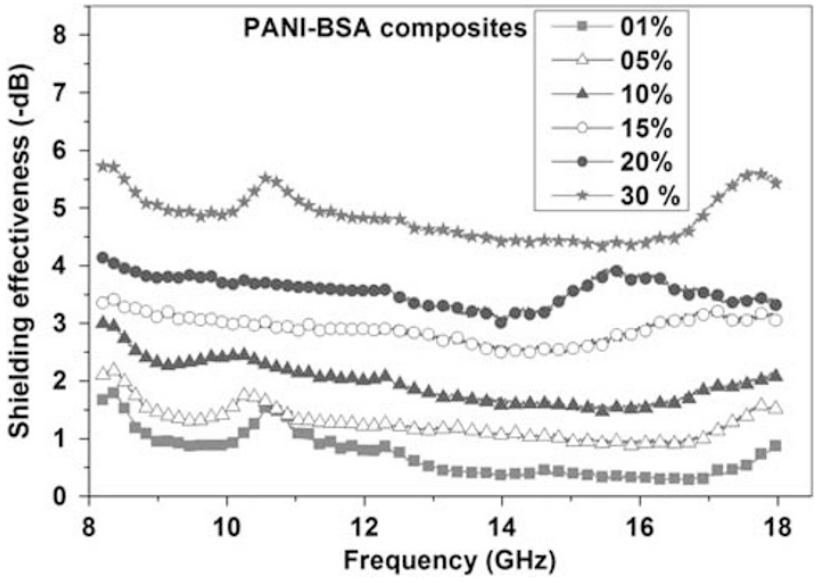

Figure 10 Shielding effectiveness curves of polyaniline/epoxy resin/hexahydrophthalic anhydride composites. PANI-BSA, polyaniline-doped benzene sulfonic acid. A full color version of this figure is available at Polymer Journal online. imperative for shifting the curing temperature to lower values to render the resulting composites more conductive.

\section{ACKNOWLEDGEMENTS}

We acknowledge the researchers of 'Département Chimie and Environnement' at Ecole des Mines de Douai, France, for experimental assistance and helpful discussions.

1 Kotsilkova, R. in Thermoset Nanocomposites for Engineering Applications (ed. Kotsilkova R.) Ch. 6, 207-276 (Smithers Rapra Technology Limited, UK, 2007). 2 Patidar, D., Jain, N., Saxena, N. S., Sharma, K. \& Sharma, T. P. Electrical properties of CdS/polyaniline heterojunction. Braz. J. Phys. 36, 1210-1212 (2006).

3 Özlem, Y., Manoj Kumar, R. \& Matt, A. in The New Frontiers of Organic and Composite Nanotechnology, (eds Victor, E., Manoj Kumar, R. and Ozlem Y.), Ch. 9, 435-475 (Elsevier, Oxford, UK, 2008).

4 Marie, A. Handbook of Conducting Polymers, 2nd edn (eds Skotheim, T.A. et al.), 921-944 (Marcel Dekker, INC, New York, 1998).

5 Poopathy, K. Curable electrically conductive resin with polyaniline fillers. Polymer $\mathbf{3 4}$, 2907-2908 (1993). 
6 Tiezhu, F., Jia, L., Jing, W. \& Hui, N. Cure kinetics and conductivity of rigid rod epoxy with polyaniline as a curing agent. Polym. Compos. 30, 1394-1400 (2009).

7 Jiongxin, L., Moon, K. S., Kim, B. K. \& Wong, C. P. High dielectric constant polyaniline/ epoxy composites via in situ polymerization for embedded capacitor applications. Polymer 48, 1510-1516 (2007).

8 Sambhu, B., Dipak, K., Nikhil, K. S. \& Joong, H. L. Progress in preparation, processing and applications of polyaniline. Prog. Polym. Sci. 34, 783-810 (2009).

9 Luiza de Castro, F. \& Mirabel Cerqueira, R. Multilayer radar absorbing material processing by using polymeric nonwoven and conducting polymer. Mater. Res. 11, 245-249 (2008).

10 Akatsuka, M., Takezawa, Y. \& Amagi, S. Infuences of inorganic fillers on curing reactions of epoxy with a boron trifuoride amine complex. Polymer 42, 3003-3007 (2001)

11 Tsotra, P., Gatos, K. G., Gryshchuk, O. \& Friedrich, K. Hardener type as critical parameter for the electrical properties of epoxy resin/polyaniline blends. J. Mater. Sci. 40, 569-574 (2005).

12 František, K. \& Jaroslava, S. Kinetics and mechanism of curing epoxy/anhydride systems. Acta Geodyn. Geometer. 4, 85-92 (2007).

13 Heise, M. S. \& Martin, G. C. Mechanism of 2-ethyl-4-methylimidazole in the curing of the diglycidyl ether of bisphenol A. J. Polym. Sci. 26, 153 (1988).

14 Dian, L., Zixing, S., Masashi, M. \& Jie, Y. DSC investigation of the hindered effect on curing behavior for epoxy-phenol/MMT nanocomposites based on the acidic octadecylamine modifier. Polymer 47, 2918-2927 (2006).

15 Heise, M. S. \& Martin, G. C. Analysis of the cure kinetics of epoxy/imidazole resin systems. J. Appl. Polym. Sci. 39, 721 (1990).

16 Belaabed, B., Lamouri, S., Naar, N., Bourson, P. \& Ould Saad, H- S. Polyaniline-doped benzene sulfonic acid/epoxy resin composites: structural, morphological, thermal and dielectric behaviors. Polym. J. 42, 546-554 (2010).

17 Rosu, D., Cascaval, C. N., Mustata, F. \& Ciobanu, C. Cure kinetics of epoxy resins studied by non-isothermal DSC data. Thermochimica. Acta 383, 119-127 (2002).

18 Mohammad, F. in Conducting Polymers, Handbook of Advanced Electronic and Photonic Materials and Devices, Vol. 8 (ed. Nalwa, H.S.) (Academic Press, London, UK, 2001).

19 Jia, W., Tchoudakov, R., Segal, E., Joseph, R., Narkis, M. \& Siegmann, A. Electrically conductive composites base on epoxy resin with polyaniline-DBSA fillers. Synth. Met. 132, 269-278 (2003)

20 Zee, R. H., Huang, Y. H., Chen, J. J. \& Jang, B. Z. Properties and processing characteristics of dielectric-filled epoxy Resins. Polym. Compos. 10, 205-214 (1989).

21 Margit, H., Jozsef, K.- K. \& Marco, H. Influence of fillers and additives on the cure kinetics of an epoxy/anhydride resin. Eu. Polym. J. 43, 1168-1178 (2007).

22 Palaniappan, S., Sreedhar, B. \& Nair Smitha, M. Polyaniline as a curing agent for epoxy resin: cure kinetics by differential scanning calorimetry. Macromol. Chem. Phys. 202, 1227-1231 (2001)

23 Pellegrino, M., Mario, A., Giuseppe, R. \& Gennaro, S. A study by Raman, near-infrared and dynamic-mechanical spectroscopies on the curing behaviour, molecular structure and viscoelastic properties of epoxy anhydride networks. Polymer 48, 3703-3716 (2007)
24 Gang, S., Hongguang, S., Yu, L., Binyuan, Z., Na, Z. \& Keao, H. Comparative study on the curing kinetics and mechanism of a lignin-based-epoxy/anhydride resin system. Polymer 48, 330-337 (2007).

25 Park, S.- J. \& Jin, F.- L. Thermal stabilities and dynamic mechanical properties of sulfone containing epoxy resin cured with anhydride. Polym. Degrad. Stab. 86, 515-520 (2004).

26 Omrani, A. \& Rostami Abbas, A. Understanding the effect of nano- $\mathrm{Al}_{2} \mathrm{O}_{3}$ addition upon the properties of epoxy-based hybrid composites. Mater. Sci. Eng. A 185-190 (2009).

27 Soto-Oviedo, Mauro A., Aráujo Olacir, A., Faez, R., Rezende Mirabel, C. \& De Paoli, Marco A. Antistatic coating and electromagnetic shielding properties of a hybrid material based on polyaniline/organoclay nanocomposite and EPDM rubber. Synth. Met. 156, 1249-1255 (2006).

28 Holloway, C. L., Hill, D. A., Ladbury, J., Koepke, G. \& Garzia, R. Shielding effectiveness measurements of materials using nested reverberation chambers. IEEE Trans. Electromagn. Compatib. 45, 350-356 (2003).

29 Kranbuehl, D. E., Delos, S. E. \& Jue, P. K. Dielectric properties of the polymerization of an aromatic polyimide. Polymer 27, 11-18 (1986).

30 Ravikiran, Y. T., Lagare, M. T., Sairam, M., Mallikarjuna, N. N., Sreedhar, B., Manohar, S., MacDiarmid, A. G. \& Aminabhavi, T. M. Synthesis, characterization and low frequency $\mathrm{AC}$ conduction of polyaniline/niobium pentoxide composites. Synth. Met. 156, 1139-1147 (2006).

31 Natsuko, N., Yu-Hsin, L., Anthony, J. B., Vivek, M. P., Chad, R. S., Steven, C. R. \& Michael, Mc B. Dielectric properties of nylon 6/clay nanocomposites from on-line process monitoring and off-line measurements. Polymer 46, 7201-7217 (2005).

32 Truong, V.- T. \& Ternan, J. G. Complex conductivity of a conducting polymer composite at microwave frequencies. Polymer 36, 905-909 (1995).

33 Navin, C. \& Deepak, J. Evaluation of a.c. conductivity behaviour of graphite filled polysulphide modified epoxy composites. Bull. Mater. Sci. 27, 227-233 (2004).

34 Psarras, G. C. Hopping conductivity in polymer matrix-metal particles composites. Composites (Part A) 37, 1545-1553 (2006).

35 Tataroglu, A., Yucedag, I. \& Altindal, S. Dielectric properties and ac electrical conductivity studies of MIS type Schottky diodes at high temperatures. Microelectron. Eng. 85, 1518-1523 (2008).

36 Chen, L. F., Ong, C. K., Neo, C. P., Varadan, V. V. \& Varadan, V. K. Microwave Electronics, Measurements and Materials Characterization (John Wiley \& Sons, London, UK, 2004).

37 Afzal, A. B., Akhtar, M. J., Nadeem, M. \& Hassan, M. M. Dielectric and impedance studies of DBSA doped polyaniline/PVC composites. Curr. Appl. Phys. 10, 601-606 (2010).

38 Sudha, J. D., Sivakala, S., Prasantha, R., Reena, V. L. \& Radhakrishnan, N. P. Development of electromagnetic shielding materials from the conductive blends of polyaniline and polyaniline-clay nanocomposite-EVA: Preparation and properties. Compos. Sci. Technol. 69, 358-364 (2009).

39 Tsotra, P. \& Friedrich, K. Electrical and dielectric properties of epoxy resin/polyanilineDBSA blends. J. Mater. Sci. 40, 4415-4417 (2005).

40 Cristiane, R. M., Roselena, F., Mirabel, C. R. \& Marco, A. D. P. Microwave absorption properties of a conductive thermoplastic blend based on polyaniline. Polym. Bull. 51, 321-326 (2004).

41 Al-Moayed, N. N., Afsar, M. N., Khan, U. A., McCooey, S. \& Obol, M. Nano ferrites microwave complex permeability and permittivity, measurements by $T / R$ technique in waveguide. IEEE Trans. Magn. 44, 1768-1772 (2008). 